\title{
A simple, repeated rTMS protocol effectively removes auditory verbal hallucinations in a single patient study
}

\author{
Maria Jose Blanco-Lopez, Javier Cudeiro-Blanco, Guillermo Iglesias, Ana Gago, Javier \\ Cudeiro
}

Based on imaging studies that showed abnormal patterns of activation in left temporo-parietal cortex (TPC) during auditory verbal hallucinations (AVH), it has been proposed that low-frequency repetitive Transcranial Magnetic Stimulation (rTMS) might be used to modify activity in the affected brain area. Following this idea Hoffman et al. (1999) first reported positive effects in a pilot study subsequently confirmed by others. However there remains some controversy and other authors have found that rTMS is no more effective than sham treatment (Slotema et al., 2011). Finally, recent reviews have shown that including of studies with larger patient samples, the effect of rTMS directed at the left temporoparietal area for AVH appear smaller, although still significant (Slotema et al., 2012 and Slotema et al., 2014).

The neurophysiological bases underlying rTMS effects are incompletely understood but may reflect changes in neural plasticity similar to long-term depression (LTD), modifying the operational properties of a given brain network. In most preliminary reports, rTMS treatment was delivered in a single 10 working day block. The question remains: would another temporal pattern of application induce stronger plastic changes? Synapses can dynamically tune their sensitivity to the incoming signals to re-set the threshold of activity required for LTD (metaplasticity) (Abraham and Bear, 1996). In other words, after a priming event, a second stimulation could improve strength and duration of effects.

With this in mind, we applied a modified version of the Hoffman's protocol (Hoffman et al., 2003) to one schizophrenic patient with AVH. An 18 year-old female was referred to our outpatient clinic by her psychiatrist. Since she was 16 , she had hopeless, severe anxiety, and internal degrading, dysphoric voice urging her to end her suffering. She was treated with antidepressants, mood stabilizers, and antipsychotics (risperidone, aripiprazole, quetiapine, amisulpride and, finally, clozapine); responses were essentially nil. Prior to rTMS her PHQ-9 score was 23, ICG 5 and PYSRATS 25. Treatment was given in two blocks. An initial course of 10 daily sessions (over two weeks) of low-frequency (1 Hz) rTMS over the left temporoparietal cortex defined as halfway between T3 and P3 (Hoffman et al., 2003) using the EEG International 10/20 System. Resting Motor Threshold (RMT) was determined daily and 1200 stimuli per session were given at $90 \%$ RMT. After one month of resting (no stimulation), we repeated the protocol. Antipsychotic medication (350 mg/day clozapine) was maintained. Informed consent was obtained from the patient before each course of rTMS treatments and the protocol complied with the University of A Coruña Ethics Committee guidelines.

During the first period effects of treatment were minimal and the voice only stopped twice (20 s at day $5,45 \mathrm{~s}$ at day 9); the amount of negative content of the voice was unchanged. During the second period of stimulation the improvement was progressive and astonishing: at day 4th AVH ceased for $1 \mathrm{~h}$, at day 6th $2.5 \mathrm{~h}$, at day 8 th $4 \mathrm{~h}$ and at day $9 \mathrm{AVH}$ stopped completely. These changes resulted in a marked reduction in the distress, anxiety, and disruption of family/social activities induced by the AVH, and an overall improvement in daily life. After treatment, the patient's mood has improved greatly (PHQ-9 = 7; ICG$S=1$ ) and she returned to study. Seven months later the patient is still free of voices and antipsychotic medication has been reduced ( $300 \mathrm{mg} /$ day clozapine).

It has been shown that repetitive transcranial magnetic stimulation efficiently reduces resistant auditory hallucinations in patients with schizophrenia but long-term impact of the treatment has been rarely reported. The case presented here encourages the use of rTMS to treat pharmacological resistant AVH. Future larger studies should examine the efficacy of protocols, perhaps conveniently distributed over time, aimed to produce stronger effects on neuroplasticity. 


\section{Conflict of interest}

We wish to confirm that there are no known conflicts of interest associated with this publication and there has been no significant financial support for this work that could have influenced its outcome.

\section{Contributors}

We confirm that the manuscript has been read and approved by all named authors and that there are no other persons who satisfied the criteria for authorship but are not listed. We further confirm that the order of authors listed in the manuscript has been approved by all of us.

\section{Acknowledgment}

We are indebted to Dr. Kenneth Grieve for his suggestions and the editing of the manuscript.

\section{References}

Abraham and Bear, 1996. W.C. Abraham, M.F. Bear. Metaplasticity: the plasticity of synaptic plasticity. Trends Neurosci., 19 (4) (1996), pp. 126-130.

Hoffman et al., 1999. R.E. Hoffman, N.N. Boutros, R.M. Berman, E. Roessler, A. Belger, J.H. Krystal, D.S. Charney. Transcranial magnetic stimulation of left temporoparietal cortex in three patients reporting hallucinated "voices". Biol. Psychiatry, 46 (1) (1999), pp. 130-132.

Hoffman et al., 2003. R.E. Hoffman, K.A. Hawkins, R. Gueorguieva, N.N. Boutros, F. Rachid, K. Carroll, J.H. Krystal. Transcranial magnetic stimulation of left temporoparietal cortex and medication resistant auditory hallucinations. Arch. Gen. Psychiatry, 60 (1) (2003), pp. 49-56.

Slotema et al., 2011. C.W. Slotema, J.D. Blom, A.D. de Weijer, K.M. Diederen, R. Goekoop, J. Looijestijn, K. Daalman, A.M. Rijkaart, R.S. Kahn, H.W. Hoek, I.E. Sommer. Can low-frequency repetitive transcranial magnetic stimulation really relieve medication-resistant auditory verbal hallucinations? Negative results from a large randomized controlled trial. Biol. Psychiatry, 69 (5) (2011), pp. 450-456.

Slotema et al., 2012. C.W. Slotema, A. Aleman, Z.J. Daskalakis, I.E. Sommer. Meta-analysis of repetitive transcranial magnetic stimulation in the treatment of auditory verbal hallucinations: update and effects after one month. Schizophr. Res., 142 (1-3) (2012), pp. 40-45.

Slotema et al., 2014. C.W. Slotema, J.D. Blom, R. van Lutterveld, H.W. Hoek, I.E. Sommer. Review of the efficacy of transcranial magnetic stimulation for auditory verbal hallucinations. Biol. Psychiatry, 76 (2) (2014), pp. 101110 